\title{
Dietary Management of Polycystic Ovarian Syndrome (Pcos)
}

\author{
Anita Mani* \\ GIFT IVF Center, Cochin, India
}

\author{
Corresponding author \\ Anita Mani, GIFT IVF Center, Cochin, India.
}

Submitted: 04 June 2017; Accepted: 10 June 2017; Published: 21 June 2017

\section{Introduction}

Polycystic Ovarian Syndrome (PCOS) is an endocrine disorder found in women, which has increasingly become common now due to the changes in lifestyle. Explanation for the increasing number of incidents can be attributed to the change in diet in most parts of the world. PCOS is found in about 10 to $20 \%$ of women depending upon the geographical location. This disorder is characterized by irregular periods, Hirsuitism and other hyper androgenic symptoms, sub-fertility and also weight gain/obesity. Life style changes with alteration in diet and exercise are effective non-pharmacological methods in restoring reproductive function in PCOS [1]. The required dietary changes will be discussed in more details in this article.

\section{Problems in Current Dietary Pattern}

If we take a look at the eating habits of the majority of the people, it can be clearly observed that there is a remarkably noticeable shift from eating fresh home cooked food to readymade, processed food on a daily basis. This diet lacks fiber, nutrients and minerals and so the amount of fiber intake has reduced drastically, especially in the developed countries. To add to the woes, there is an increased use of preservatives, chemicals, pesticides, chemical fertilizers from the initial stages of farming till the goods are packed and sold in the markets. Abundant use of plastics has also resulted in food and water contamination. In different ways, many chemicals, which are harmful and disrupt endocrine system, are getting into the human system. The general calorie intake has also shot up over the last couple of decades and this factor along with reduction in physical activities is causing the metabolic syndrome which in turn leads to PCOS. Exercise and diet can alter the metabolic syndrome associated with PCOS [2].

\footnotetext{
About Micro Biome

When we look at the alterations required in the subject of nutrition, there are very clear recommendations coming from the various researches happening worldwide. The five-year human micro biome project launched in 2008 has shed new light on the role of micro biome on human physiology. Trillions of microorganisms live on human body surface, eyes, mouth and gut area. The combined genomes of this micro biota and human body are micro biome, which determines the health of a person. Changes in lifestyle with reduction in fiber and increased consumption of processed food has led to alteration in of micro biome in our body, so we have a different set of bacteria compared to our predecessors and the body is less protected against many of the inflammatory disorders. This has an adverse impact on digestion and all the body functions including endocrine system and the central nervous
}

system is affected by this change in micro biome. To improve the health condition, we should acquire back the lost good bacteria and for this we need to change our diet, so that we don't harm the good bacteria and provide them a favorable environment to grow back. Fiber rich food like raw banana, fresh fruits and salads, along with plenty of green leafy vegetables are good for the gut bacteria. When we feed the good bacteria their number will increase exponentially and this will help in controlling the metabolic syndrome.

\section{Role of Insulin Resistance}

Another characteristic found in most of the women with PCOS is high amount of insulin in blood and insulin resistance [3]. The high intake of processed sugar and processed carbohydrates increases insulin level (hyperinsulinemia) and with high fat intake cells will become resistant to insulin. When there is high level of insulin, blood sugar is not entering the cells/tissue and there is profound fatigue felt, hence lethargy is very common in PCOS just like in diabetes mellitus. To prevent this from happening, ideally women with PCOS should reduce the carbohydrate intake; consume more complex carbohydrates and food with low glycemic index. For example, whole wheat is always better than refine flours, brown rice should be preferred over white rice and oats, millets and other grains, which are soluble fibers, should be included in the diet, but taken moderately. When it comes to problems related to insulin, we tend to forget the role of lipids. All the researches are showing that there is central obesity and lipocyte dysfunction during the condition of PCOS and metabolic syndrome [4]. Lipids play a very important role in the management of diet. Total amount of fat intake should be drastically reduced, trans fat should be avoided, fried food/oily food must be taken rarely only if unavoidable. Dairy products and animal foods should be minimized, generally fat found in plant food source are in much better form and less in content. This way the insulin resistance can be reduced, weight reduction can be achieved, metabolic rate can be improved and patients can control the conditions like acne, excessive hair symptoms, skin disorders and other psychological problems associated with high androgen levels [5,6]. Psychological problems due to the high level of androgen like anxiety, depression, mood variation, relationship problems, social isolation etc can be reduced by reduction in the fat intake as it reduces level of androgens [7].

\section{Modification in Eating Pattern}

What we eat, how we eat and when we eat plays a very important role in controlling PCOS. Ideally breakfast should be taken within 30 minutes of waking up, so that the metabolic system kick starts and sufficient energy is released. When there is delay in taking 
breakfast, the basal metabolic rate becomes slow, the cells do not release energy, hence the person tends to feel tired and lethargic and may also experience headache while doing his/her work. Throughout the day, small portions of food should be taken at regular intervals, i.e. in every four to five hours you should eat something to keep your metabolism going [8]. Large portions of food will result in high level of insulin release in the blood which may cause insulin resistance. Breakfast should be complete and form the most important meal of the day and should include complex carbohydrates, proteins, plenty of vitamins and minerals in the form of fresh fruits. Lunch should be lighter than breakfast and taken on time without much delay. The dinner or the last meal should be eaten two to three hours before sleeping and should be minimal as possible. Now a day's people tend to eat food in haste due to the hectic schedule, which is a very bad habit as it affects the process of digestion. The digestion process starts in the mouth, so food should be chewed properly and eaten slowly. It takes time for our brain to register that stomach is full, so if you eat fast you will end up eating more than what is required and put on weight. Also studies show that if you enjoy the process of eating you feel more satisfied and tend to eat less.

\section{Role of Diet in Chronic Inflammation in PCOS}

Many PCOS patients suffer from chronic inflammations like inflammable bowel disease and appropriate management of the diet by giving attention to timing, nourishment and quantity will definitely help in controlling the bowel disorders [9]. Almost all the systems in the body are affected due to the inflammatory changes happening due to PCOS and even the gums can get inflamed causing gingivitis. Keeping the sugar intake low and practicing good dental hygiene will help control/avoid gingivitis. As the immunity is reduced, women with PCOS are more prone to infections like common cold or candidiasis and other skin infections. If the intake of processed sugar in form of sweets, cake, biscuits and other deserts or processed food is avoided, immunity can be improved. Women with recurrent candidiasis should avoid foodstuff like cakes, doughnuts and bread, which contains yeast. Many herbs and fruits are rich in vitamins and adding plenty of herbs to the food will not only make it look good and taste good but help build the body immunity. Many tropical fruits like Indian gooseberry/Amla is very rich in vitamin $\mathrm{C}$ and can aid in preventing the immunity problems found in PCOS. The high amount of antioxidants found in berries will facilitate the reduction of oxidants formed in the body especially due to lipid dysfunction.

\section{Cooking and Culinary Methods}

It is not only what we eat, but how we eat also makes a huge difference in controlling PCOS, hence the cooking method is also equally important. It is always better to boil or steam the vegetables rather than fry or barbeque. In order to make food tasty and appealing vegetables of different texture and color can be added and garnished with herbs. Throwing in some nuts and dry fruits can not only add crunch and zing, but also increase the nutritional value.

\section{Shopping Techniques}

It is not a difficult or laborious task to eat healthy. Let us look at some practical methods, which can be adapted to make our lifestyle healthy and enjoyable. The first step should start from changing the way we do shopping [10]. Next time you hit a supermarket, directly head towards the organic fresh section and fill your basket with lots of greens, different colored vegetables because they are very nutritious. Second stop should be to purchase whole grains and also pulses, which are high in fiber and has good lactobacillus content. The pulses can be soaked and germinated into sprouts which has more vitamins and nutritional value than the pulses and are easily digestible. Sprouts add crunch and taste to salads and can be consumed fresh without cooking, where as pulses needs cooking, as they are difficult to digest.

Try to skip the pastry, biscuits and bakery section completely; ignoring their existence in the supermarket might help. Frozen berries are a very good alternative to fresh berries when they are not in season. Most of the good nutrients are preserved in the frozen form and it can be a cheaper substitute to the fresh berries. The intake of diary, poultry and other animal products should be restricted and try to take them as minimum as possible. If you are a strong advocator of non-vegetarian food, cook at home during the weekends. It is a much healthier and cheaper alternative to eating out. Reducing eating out will save you money and if your start practicing this regularly you will notice that your overall diet starts becoming healthy. In a way storing lots of greens and vegetables at home can help as you tend to cook and eat what you have at home. Another advantage of cooking at home is that there is going to be fewer orders of fast food items like pizza, burgers or fries and this saves your bills as well.

\section{Summary}

To sum up, these changes in the way one eat should help to get good control over the problems related to polycystic ovaries. Research has proven that even a 5\% weight loss can regularize/ bring back the periods and a good diet will definitely help in achieving the goal of reproduction. Proper consultation with the nutritionist and fitness expert can actually make a difference and avoid numerous trips to doctors of different specialties. In the long run PCOS patients often end up visiting a lot of doctors starting from a general practitioner to a Dermatologist for cosmetic problems, a Gynecologist for irregular periods, Infertility specialist for sub fertility, Diabetologist for diabetes Mellitus, Cardiovascular specialist for cardiac problems like hypertension and cardiovascular accidents, Psychiatrist for psychological issues like anxiety and depression and so on. In order to avoid these frequent visits and to get control of PCOS, the best step will be to learn in detail about nutrition and visit an expert dietitian.

The author Dr. Anita Mani is a practicing infertility specialist from south India with special interest in PCOS.

\section{Reference}

1. Huber-Buchholz MM, Carey DG, Norman RJ (1999) Restoration of reproductive potential by lifestyle modification in obese polycystic ovary syndrome: role of insulin sensitivity and luteinizing hormone, J Clin Endocrinol Metab 84: 1470-1474.

2. Barnard RJ, Wen SJ (1994) Exercise and diet in the prevention and control of the metabolic syndrome. Sports Med 18: 218-228.

3. Legro RS, Finegood D, Dunaif AA (1998) fasting glucose to insulin ratio is a useful measure of insulin sensitivity in women with polycystic ovary syndrome, J Clin Endocrinol Metab 83: 2694-2698.

4. Lord J, Thomas R, Fox B, Acharya U, Wilkin T (2006) The central issue? Visceral fat mass is a good marker of insulin resistance and metabolic disturbance in women with polycystic ovary syndrome, BJOG 113: 1203-1209.

5. Kiddy DS, Hamilton-Fairley D, Bush A, Frances Short, Vtctor 
Anyaoku, et al. (1992) Improvement in endocrine and ovarian function during dietary treatment of obese women with polycystic ovary syndrome. Clin Endocrinol (Oxf) 36: 105-111.

6. Pasquali R, Antenucci D, Casimirri F, Venturoli S, Paradisi R, et al. (1989) Clinical and hormonal characteristics of obese amenorrheic hyperandrogenic women before and after weight loss. J Clin Endocrinol Metab 68: 173-179.

7. Adali E, Yildizhan R, Kurdoglu M, Kolusari A, Edirne T, et al. (2008) The relationship between clinico-biochemical characteristics and psychiatric distress in young women with polycystic ovary syndrome, J Int Med Res 36: 1188-1196.
8. Willett W (1998) Food Frequency Methods, Nutritional Epidemiology 5.

9. Chris CJ Kelly, Helen Lyall (2001) Low grade inflammation in women with Polycystic Ovarian syndrome. J Clin Endocrinol Metab 86: 2453-2455.

10. Kallehave O, Skov MB, Tiainen N (2010) Reducing the Paradox of Choice: Designing a Nutritious Persuasive Shopping Trolley. In Hasle et al. (eds.) Poster Proceedings of the 5th International Conference on Persuasive Technology (Persuasive 2010), Oulu University Press 25-28.
Copyright: (02017 Anita Mani. This is an open-access article distributed under the terms of the Creative Commons Attribution License, which permits unrestricted use, distribution, and reproduction in any medium, provided the original author and source are credited. 